\title{
Mi asma y yo. Relato biográfico
}

\author{
My asthma and me. Live history
}

\section{Minha asma e eu. Relato biográfico}

Raquel Asunción Díaz Alonsoํㄹ David Palacios Martínez², Pedro Adalia Serrano ${ }^{3}$

${ }^{1}$ Diplomada Universitaria en Enfermería. Hospital Universitario de Fuenlabrada. Fuenlabrada. Madrid.

${ }^{2}$ Médico de Familia. Servicio Madrileño de Salud - SERMAS. Dirección Asistencial Sur de Madrid. C.S. San Blas (Parla, Madrid). Centro de Salud San Blas. Parla. Dirección Asistencial Sur de Madrid.

${ }^{3}$ Psicólogo. Miembro del EuroPsy. Coordinador del Gabinete Aguirre. C/Aguirre, 1, 2 a planta. Madrid. Cómo citar este artículo en edición digital: Díaz Alonso, R.A., Palacios Martínez, D. y Adalia Serrano, P. (2015). Mi asma y yo. Relato biográfico. Cultura de los Cuidados (Edición digital), 19, 41. Disponible en: http://dx.doi.org/10.14198/cuid.2015.41.03>

Correspondencia: Raquel Asunción Díaz AlonsoDirección postal: calle Nazaret, nº6, portal 2, 2B, 28980, Parla (Madrid). Correo electrónico: raquelda_parla@hotmail.com Recibido: 11/12//2014; Aceptado: 17/03/2015

Para la elaboración del presente trabajo no se han recibido ningún tipo de becas ni de soporte financiero. Así mismo, los autores declaran que no existe ningún tipo de conflicto de intereses en la elaboración del presente trabajo.

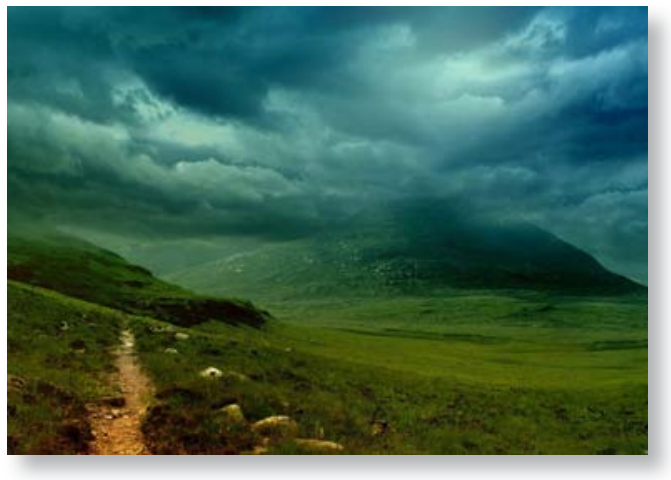

\section{ABSTRACT}

The asthma is a disease which is characterized by the intermittent airway obstruction to the air-flow. Its world prevalence ranges between $1-24 \%$. It courses clinically with dyspnea, cough, wheezing and/or chest pain.

The degree of adherence to the treatment and disease control are influenced by the acceptance of the disease by the patients, their knowledge about it, and their strategy of coping with the asthma.

This paper aims to provide a holistic and interdisciplinary point of view of the disease. We have conducted an interview in depth and a history of life of Daniel, our informer. We have collected and interpreted his experience of the disease with biographic and descriptive characteristic.

Keywords: asthma; bronchial diseases; qualitative research; holistic health; interdisciplinary research.

\section{RESUMO}

A asma é uma doença crônica caracterizada por obstrução intermitente das vias aéreas ao fluxo de ar. Em todo o mundo prevalência varia entre 1-24. Ele é clinicamente caracterizado pelo aparecimento de falta de ar, tosse, chiado eu aperto no peito.

A aceitação da doença, conhecimento e pelos pacientes e suas famílias lidar estratégia influencia o grau de adesão ao tratamento e controle da asma.

O presente trabalho tem como objetivo proporcionar uma doença holística e interdisciplinar. Através da entrevista em profundidade e o registo biográfico de Daniel, nosso informante, temos coletado e jogado com caráter biografico-descriptivo sua experiência da doença. 
Palavras-chave: asma; broncopatias; pesquisa qualitativa; saúde holística; pesquisa interdisciplinar.

\section{RESUMEN}

El asma es una enfermedad crónica caracterizada por la obstrucción intermitente de las vías respiratorias al flujo aéreo. Su prevalencia a nivel mundial oscila entre 1-24\%. Clínicamente se caracteriza por la aparición de disnea, tos, sibilancias y/o opresión torácica.

La aceptación de la enfermedad, su conocimiento y la estrategia de afrontamiento por parte de los pacientes y sus familias influye en el grado de cumplimiento terapéutico y de control del asma.

El presente trabajo pretende aportar una visión holística e interdisciplinar de la enfermedad. A través de la entrevista en profundidad y el registro biográfico de Daniel, nuestro informante, hemos recogido e interpretado con carácter biográfico-descriptivo su vivencia de la enfermedad.

Palabras clave: asma; enfermedades bronquiales; investigación cualitativa; salud holística; investigación interdisciplinaria.

\section{INTRODUCCIÓN}

El asma es una enfermedad crónica caracterizada por la obstrucción intermitente de las vías respiratorias al flujo aéreo. Aparece como respuesta a diferentes estímulos. Se asocia a hiperreactividad bronquial. Cursa de manera episódica, recurrente y oscilante. Actualmente es imposible predecir la evolución del asma. (Seguí, 2004; Ancoechea, 2006; GINA, 2006; GEMA, 2009; Chávez, 2012).

Afecta a alrededor de 300 millones de personas en todo el mundo. Su prevalencia oscila entre el 1-24\% según el país y el estudio con- sultado. Una de cada 250 muertes en el mundo es debida al asma. En España afecta al 1-6\% de los niños y al 4-8\% de los adultos. Produce una importante carga social y económica, ausentismo escolar y laboral, limitación de las actividades físicas y de localidad de vida, etc. (Seguí, 2004; Ancoechea, 2006; GINA, 2006; GEMA, 2009; Chávez, 2012).

La etiología del asma es multifactorial y su fisiopatología es compleja. Está influenciada por múltiples factores: susceptibilidad individual, cierta carga genética no bien conocida actualmente, factores intrínsecos, ambientales y psicológicos. (Seguí, 2004; Ancoechea, 2006; GINA, 2006; GEMA, 2009; Chávez, 2012).

El asma suele debutar en la infancia, aunque puede hacerlo a cualquier edad de la vida. Suele disminuir con la edad. La clínica característica del asma no es específica. Consiste en disnea, tos, sibilancias y opresión torácica de predominio nocturno. Estos síntomas pueden no presentarse juntos, o desencadenarse por diferentes estímulos. Suele revertir espontáneamente o con tratamiento (Seguí, 2004; Ancoechea, 2006; GINA, 2006; GEMA, 2009; Chávez, 2012).

El diagnóstico de sospecha es eminentemente clínico. Debe confirmarse la presencia de obstrucción reversible al flujo aéreo mediante diversas pruebas: espirometría con prueba broncodilatadora, pruebas funcionales completas, medición seriada del flujo espiratorio máximo, estudios analíticos, etc. Los principales diagnósticos de enfermería relacionados con el asma están dirigidos a la educación, la promoción para la salud, el afrontamiento del estrés y al manejo terapéutico (Seguí, 2004; Ancoechea, 2006; GINA, 2006; GEMA, 2009).

El tratamiento está dirigido a controlar la enfermedad en el menor tiempo posible y a prevenir las posibles complicaciones. Es acon- 
sejable realizar un enfoque multidisplinar y biopsicosocial. En función de la gravedad del asma, el tratamiento farmacológico puede ser autoadministrado por el propio paciente, o incluso precisar medicación intravenosa o soporte ventilatorio asistido. Los fármacos antiasmáticos incluyen fármacos agonistas $\beta$-adrenérgicos de acción corta y/o prolongada, corticoides inhalados o sistémicos, y otros fármacos (oxígeno, anti-leucotrienos, etc). $\mathrm{La}$ vía inhalatoria produce menos efectos adversos, resultando rápida y eficaz. (Seguí, 2004; Ancoechea, 2006; GINA, 2006; GEMA, 2009; Chávez, 2012; González, 2014).

Para comprender y manejar de una manera holística al paciente asmático es importante valorar también su aspecto psicológico. La disnea del asma puede originar o incrementar la ansiedad tanto en niños como en adultos. La percepción adecuada de la disnea por parte de los pacientes está basada en su propia experiencia y en el aprendizaje. La ansiedad es un agravante del asma, estando ambos vinculados. (Chávez, 2012; González, 2014).

Las técnicas respiratorias y de relajación más utilizadas en psicoterapia ayudan considerablemente a los pacientes asmáticos. Así lo demuestran diversos estudios científicos, especialmente durante las últimas dos décadas. Dichas técnicas resultan más efectivas cuando se practican con regularidad. De este modo, ayudan a controlar la ansiedad y la angustia durante las crisis agudas y en los momentos posteriores, donde podrían producirse respuestas de evitación y pánico. Las técnicas más aconsejables son respiración abdominal y relajación creativa. Pueden aprenderse en muy pocas sesiones. El paciente debe practicarlas posteriormente en su domicilio o en su lugar de trabajo, creando un hábito saludable para afrontar con mayor control y calma su dolencia.
El asma puede tener un impacto considerable sobre la familia, especialmente cuando afecta a un niño. En ocasiones, llega a constituir el centro de su preocupación, cambiando la vida, la conducta y la dinámica familiar. La familia puede intentar sobreproteger al niño, limitando su autonomía y fomentando su dependencia (Chávez, 2012).

La educación sanitaria al paciente asmático y su familia por parte de todos los profesionales es fundamental. Facilita la comprensión de la enfermedad, la implicación y el empoderamiento del paciente sobre su enfermedad y su tratamiento. De este modo, mejora la autoeficacia y el control de la enfermedad y disminuyen la comorbilidad y las complicaciones.

A continuación presentamos el relato biográfico de Daniel, un paciente diagnosticado de asma en su infancia. En el relato nos explica su enfermedad, su vivencia de los síntomas, cómo las crisis de asma afectan a su vida y a la calidad de la misma. Daniel, al comienzo de la entrevista, manifiesta expresamente su interés en dar a conocer su historia. Piensa que podría ayudar a otras personas.

La información fue recogida mediante la realización de una entrevista abierta en profundidad, desarrollada en el domicilio particular del informante. Su duración aproximada fue de una hora y media. Previo consentimiento informado, grabamos la entrevista en formato de audio, recogiendo algunas además algunas notas de campo. Los datos recopilados fueron transcritos literalmente y sometidos a un proceso de análisis de datos cualitativos de acuerdo a los pasos descritos por Amezcua y Hueso, y por Martín Muñoz y sus colaboradores (Amezcua, 2009; Martín, 2013). Para conservar la confidencialidad de nuestro paciente, hemos utilizado nombres ficticios en la reconstrucción del relato biográfico. 


\section{BIBLIOGRAFÍA}

- Amezcua, M. y Hueso, C. (2009). Cómo analizar un relato biográfico. Archivos de la Memoria [online], (6 fasc.

3). Disponible en: http://www.index-f.com/memoria/6/ mc30863r.php

- Ancoechea Bermúdez, J. y Ricote Belinchón, M. (2006). Proceder. Procedimientos en enfermedades respiratorias. Asma. Barcelona: Ed. Grupo Ars XXI de Comunicación S.L.

- Chávez Ventura, G. (2012). Perspectivas para la investigación psicológica del asma. Revista Psicológica Trujillo, 14(2), 268-277.

- Global Initiative for Asthma (2006). GINA 2006. Global Initiative for Asthma. Global Strategy for Asthma Management and Prevention NHBLI/WHO Workshop Report. Disponible en: http://www.ginasthma.com

- González Suárez, M. y Duarte Clíments, G. (2014). Terapia cognitivo-conductual y enfermería. A propósito de un caso. ENE enf, 8(1), Disponible en: http://eneenfermeria.org/ojs/index.php/ENE/article/view/295

- Guía española del manejo del asma (2009). GEMA (Guía española del manejo del asma). Archivos de Bronconeumología,45(7), 2-35.

- Martín Muñoz, B., Gálvez González, M. y Amezcua, M. (2013). Cómo estructurar y redactar un Relato Biográfico para publicación. Index de Enfermería, 22(12), Disponible en: http://dx.doi.org/10.4321/S113212962013000100018.

- Seguí Díaz, M., Linares Pou, L. y Ausín Olivera, A. (2004). El asma bronquial desde el médico de familia (I). Semergen, 30(10), 514-523.

\section{RELATO BIOGRÁFICO.}

\section{Dando guerra desde que nací.}

Me llamo Daniel. Soy atópico y asmático. La atopía afecta a mi piel con brotes frecuentes de dermatitis y me produce alergia al polen por temporadas. El asma me produce crisis respiratorias con cierta frecuencia. Quizás pueda parecer un problema anodino. Sin embargo, afecta a mi vida y la de los que me rodean.
Según cuentan mis padres, he padecido estas enfermedades desde siempre. Antes de cumplir mi primer mes de vida, sufrí mi primera crisis de asfixia. Ellos se asustaron muchísimo. Recuerdan que, más o menos de repente, comencé a respirar con gran dificultad. Se me amorataron los labios. Se me marcaban mucho las costillas y, también parecía que respiraba moviendo el abdomen. Debió ser una experiencia traumática para mis padres, porque hoy en día, 37 años después, todavía se les nota algún gesto de terror cuando recuerdan aquellas hazañas...

Nunca olvidan su angustia al tener que llevarme en esas condiciones al hospital. La espera interminable, agónica y pavorosa mientras me valoraban y trataban aquellas crisis. La alegría al ver que respondía al tratamiento y que habían logrado salvar mi corta vida. Y, tras recibir el alta médica, el miedo a las recaídas. Las noches de insomnio con los ojos y los oídos abiertos por si aparecía una nueva crisis... Nuevas crisis, que volvieron a aparecer, acompañándome con cierta frecuencia y gravedad variable durante toda mi infancia.

\section{Respirar parece tan sencillo...}

Si te lo planteas, respirar parece una tontería. Ni siquiera hay que pensar en ello. Se hace de manera inconsciente. Los pulmones lo hacen por sí mismos. Pero en esos momentos, en una crisis de asma, no quieren. Supongo que en realidad no pueden. Afortunadamente, el cuerpo sabe qué hacer: intentar respirar con más fuerza todavía.

\section{Lo normal no es asfixiarse.}

Entre crisis y crisis, yo era un niño normal. Jugaba, corría o estudiaba como los demás niños sanos. Sin embargo, cuando me sorprendía una crisis respiratoria volvíamos al 
mismo ciclo de siempre: aparecía la sensación de asfixia, me costaba respirar, dejaba de jugar o hacer las cosas que estuviese haciendo, me trataban con inhaladores; y después, cuando por fin la respiración volvía a la normalidad, la acompañaba un cansancio extenuante.

Podríamos decir que esta era "mi rutina". Como para mí siempre había sido así, yo lo veía normal. Suponía que nos pasaba a todos los niños.

Para mi sorpresa, en el colegio descubrí que al resto de niños no les pasaba lo mismo. Ellos no se asfixiaban cada dos por tres.

Al sentirme diferente a los demás niños, me veía a mí mismo "especial para mal". Algunas veces me cuestionaba “ipor qué yo me asfixio y los otros niños no?”. Incluso llegué a preguntárselo a mis padres. Pobrecillos. Qué difícil debe de ser explicarle estas cosas a un niño.

\section{Mis padres, un pilar fundamental.}

Con el paso del tiempo, mis padres aprendieron a reconocer las crisis de asma precozmente y actuar de la manera adecuada. No puede imaginarse lo agradecido que les estoy por ello. Sus caras cuando recuerdan y me explican estos logros muestran mucha más tranquilidad que cuando me hablan de las primeras crisis. Ellos mismos trataron muchas, las más leves, poniéndome en casa los inhaladores que los médicos les habían pautado.

\section{El "selecto" club de asmáticos atópicos.}

Tras varios ciclos de crisis-susto-tratamiento-extenuación-recuperación-normalidad, en algún momento de mis primeros años de vida me diagnosticaron asma.

$\mathrm{Al}$ parecer existen varios tipos de asma. Tras varias consultas por diferentes especialistas, al final llegaron a la conclusión de que mi problema respiratorio era de tipo alérgico y atópico.

El alergólogo que me diagnosticó, en torno a los 5-7 años, les explicó a mis padres que yo padecía asma persistente atópica. ¡Qué bien! Ya pertenecía al "selecto" club de pacientes que son atópicos. La atopía engloba tres enfermedades que también me pasaban: el asma, la polinosis o fiebre del heno (la "típica alergia de primavera" con molestias en los ojos, la nariz y la garganta), y la dermatitis atópica (unas lesiones rojas que me salían en la piel de vez en cuando y que picaban a rabiar). Aquel amable doctor les explicó a mis padres con voz grave, que sonaba a importante: "verán, la atopía es como una forma de ser. Igual que hay gente con los ojos azules o verdes, los pacientes atópicos son irritables: hay muchos estímulos físicos o ambientales que pueden irritarles, y producirles crisis respiratorias, de alergia al polen, o en la piel". No sé por qué se grabaron aquella escena y aquella frase en mi mente. Pero cada vez que uno de esos estímulos a los que se refería aquel doctor "me irrita” y me provoca alguna crisis, vuelvo a revivirla.

\section{Nuestro acuerdo tácito.}

Durante mi adolescencia no recuerdo ninguna crisis asmática importante. Sé que no es así, pero me gusta pensar que mi asma y yo llegamos al acuerdo tácito de no molestarnos. De hecho, he podido hacer una vida activa normal y corriente como el resto de la gente sana. Incluso me dieron el alta los neumólogos que realizaban mi seguimiento periódico, dejándole el control del asma a mi Médico de Familia.

\section{El asma ha vuelto a mi vida.}

Desde hace unos meses, el asma ha vuelto a mi vida. Con mayor intensidad. Con una sen- 
sación muy desagradable. En la primavera, tras unos días con un catarro más o menos leve, de repente un día comencé a fatigarme y a escuchar "pitos" al respirar. Tras consultar con mi médico, volvimos a la rutina de las crisis: la angustia de si habrá más crisis y cuándo, los ciclos de tratamiento, llevar siempre encima un inhalador de salbutamol por si acaso, estar pendiente e intentar evitar los estímulos que me provocaban las crisis (sobre todo el polen), etc.

En esta ocasión el tratamiento habitual no fue suficiente, a pesar de cumplirlo escrupulosamente. Súbitamente, unos días después empezó a costarme mucho respirar. Notaba una fuerte presión en el pecho que no me dejaba entrar el aire, por más fuerte que yo inspirase. Empezaron a notarse mucho mis costillas, y también mis clavículas. Mis labios volvieron a coger este tono azulado que tantas veces han tenido. Mi cuerpo comenzó a perder fuerzas. Seguir respirando me costaba un esfuerzo titánico, sobrehumano. Debí sentarme por la extenuación. Ahora, además, parecía que estaba respirando desde mi tripa. El asma había vuelto a mi vida.

En estos momentos, en plena crisis de asma, ponerse nervioso es muy sencillo. Respiras con gran dificultad y esfuerzo. Mucho esfuerzo. La sensación de asfixia angustiante. Y además la asfixia, en mayor o menor intensidad, es real.

Sin embargo, es muy importante intentar evitar la ansiedad en plena crisis de asma. Porque la sensación de asfixia empeora. Mucho. Y además, los nervios te dificultan empezar lo más precozmente posible el tratamiento inhalador. De hecho, con tranquilidad y utilizando adecuadamente los inhaladores he logrado controlar muchas crisis de asma leves en mi vida. Sin embargo, en esta ocasión no fue suficiente.
Afortunadamente para mí, mi pareja es enfermera. A pesar de que nunca antes me había visto sufrir una crisis de asma semejante, supo reconocer los síntomas. Yo, como puede suponerse, no podía hablar. Me llevó a urgencias, donde me administraron un tratamiento más intenso: medicación nebulizada, corticoides intramusculares (¡cómo duelen!), oxígeno para respirar, etc.

Tras unas horas de tratamiento en Urgencias, volví a respirar... más o menos. Los médicos lograron controlar la crisis de asma y volver a normalizar mi respiración. Me ajustaron el tratamiento y me dieron de alta. Debería tomar medicación durante unas semanas, volver a los controles precisos por parte de los médicos, etc. Diferentes profesionales (médicos, enfermeras, psicólogos, etc) ampliaron mi formación sobre la enfermedad y mi implicación en mi propio tratamiento.

\section{El asma ha amputado mi vida.}

Las primeras horas después de ser dado de alta, pude descansar en casa. Me encontraba extenuado por el esfuerzo físico de la crisis. Incluso pude dormir algo. Después de aquel descanso inicial volví a descubrir cuánto puede limitar mi vida una crisis de asma. Actividades tan simples como levantarme del sillón para ir al baño, estar de pie un ratito, hacer una cama o mantener una conversación de más de cuatro o cinco frases seguidas me dejan fatigado y extenuado. Volver a recuperar la normalidad en la respiración, y que desaparezca la fatiga me cuesta alguna hora de estar sentado en el sofá, semi-inclinado (porque parece que se respira mejor en esa posición). Sin hacer ninguna otra cosa que respirar. Incluso sin poder hablar. Ni pensar con claridad. Con este panorama, no debe resultar difícil imaginar la esfera sexual. Totalmente 
anulada. En algún momento piensas: "me van a cambiar por dos de veinte años".

Actualmente vivimos en un tercer piso, sin ascensor. En esta situación, llevo alguna semana sin poder salir de casa: subir o bajar la escalera me resulta imposible. Incluso el médico de familia viene a valorarme a mi domicilio. Tampoco puedo ir a trabajar, por lo que además se añaden todos los problemas y complicaciones derivados de la baja laboral.

Además está la medicación. En total, varios inhaladores y siete pastillas al día durante varias semanas. Lo que peor llevo son las pastillas de prednisona. Tienen un sabor muy amargo. Da la sensación de que se queda pegada a la garganta, recordándote su sabor durante todo el día. Creo que padezco muchos de sus efectos secundarios que aparecen en los prospectos. Me noto constantemente como eufórico o intranquilo, incluso irritable. Pero sin energía, porque como no respiro bien todavía, me extenúo con gran facilidad. Y además, tengo un apetito voraz e insaciable. La sensación es muy parecida al hambre. Pero, a diferencia de ésta, nunca se sacia. Por mucho que comas. Por eso llamo "Gordisona” a la prednisona.

\section{Y entonces recapitulas.}

Tengo 37 años. Soy una persona activa, con una vida normal. Pero llevo varias semanas to- mando más medicación incluso que mi abuela. Padeciendo casi todos los efectos secundarios que anuncian los prospectos. Sin poder salir de casa porque no puedo subir o bajar la escalera. Hasta ir al baño me fatiga y extenúa. No puedo concentrarme en ninguna actividad, ni leer, ni ver una película, ni ayudar en casa. Sólo puedo pensar en respirar. La sensación es esa: el asma ha amputado mi vida.

\section{Respeto, pero no miedo.}

Confío que con el tratamiento adecuado, reposo, y paciencia, mucha paciencia, la crisis desaparecerá y podré volver a mi vida habitual.

Un antiguo proverbio, creo recordar que budista, reza "el dolor es inevitable, pero el sufrimiento es opcional". Me siento muy identificado con él. Ahora que el asma y yo hemos vuelto a reencontrarnos, no puedo evitar que limite mi vida. Pero no pienso permitir que me la amargue.

No sé cuándo terminará esta crisis. No sé si después volverán otras, aunque probablemente lo harán. No puedo controlar cuándo ni dónde me sorprenderán. Pero, si las crisis de asma vuelven, estaré preparado. Y todos los que me rodean también.

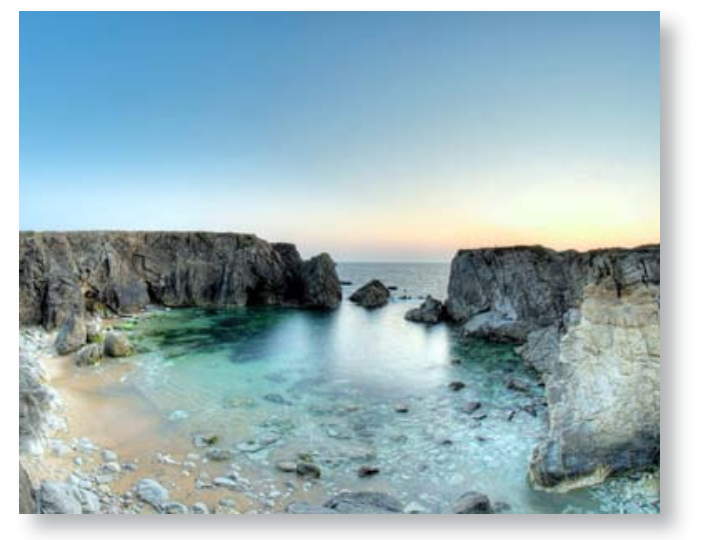

